\title{
pak2a mutations cause cerebral hemorrhage in redhead zebrafish
}

\author{
David A. Buchner*t, Fengyun Su, Jennifer S. Yamaoka*, Makoto Kamei§, Jordan A. Shavit", Linda K. Barthell, \\ Beth McGee*, Julio D. Amigo**, Seongcheol Kim ${ }^{\dagger+}$, Andrew W. Hanosh ${ }^{\ddagger}$, Pudur Jagadeeswaran ${ }^{\dagger+}$, Daniel Goldman $^{\ddagger \ddagger}$, \\ Nathan D. Lawson**, Pamela A. Raymondl, Brant M. Weinstein§, David Ginsburg*‡§§, and Susan E. Lyons ${ }^{\ddagger \uparrow \uparrow}$
}

\begin{abstract}
*Howard Hughes Medical Institute and Life Sciences Institute, ${ }^{\ddagger}$ Department of Internal Medicine, "Department of Pediatrics, IDepartment of Molecular, Cellular, and Developmental Biology, ${ }^{\ddagger \neq}$ Molecular and Behavioral Neuroscience Institute and Department of Biological Chemistry, and ११Division of Hematology/Oncology and Cellular and Molecular Biology Program, University of Michigan, Ann Arbor, MI 48109; §Laboratory of Molecular Genetics, National Institute of Child Health and Human Development, National Institutes of Health, Bethesda, MD 20892; ${ }^{\dagger}$ Department of Biological Sciences, University of North Texas, Denton, TX 76203; and **Program in Gene Function and Expression, University of Massachusetts Medical School, Worcester, MA 01605
\end{abstract}

Edited by Napoleone Ferrara, Genentech, Inc., South San Francisco, CA, and approved July 19, 2007 (received for review February 1, 2007)

The zebrafish is a powerful model for studying vascular development, demonstrating remarkable conservation of this process with mammals. Here, we identify a zebrafish mutant, redhead $\left(r d^{m i 149}\right)$, that exhibits embryonic CNS hemorrhage with intact gross development of the vasculature and normal hemostatic function. We show that the rhd phenotype is caused by a hypomorphic mutation in p21-activated kinase 2a (pak2a). PAK2 is a kinase that acts downstream of the Rho-family GTPases CDC42 and RAC and has been implicated in angiogenesis, regulation of cytoskeletal structure, and endothelial cell migration and contractility among other functions. Correction of the Pak2a-deficient phenotype by Pak2a overexpression depends on kinase activity, implicating Pak2 signaling in the maintenance of vascular integrity. Rescue by an endothelial-specific transgene further suggests that the hemorrhage seen in Pak2a deficiency is the result of an autonomous endothelial cell defect. Reduced expression of another PAK2 ortholog, pak2b, in Pak2a-deficient embryos results in a more severe hemorrhagic phenotype, consistent with partially overlapping functions for these two orthologs. These data provide in vivo evidence for a critical function of Pak2 in vascular integrity and demonstrate a severe disease phenotype resulting from loss of Pak2 function.

$\beta$-pix | CNS | endothelial cell | p21-activated kinase | vasculature

A monolayer of vascular endothelial cells regulates the barrier between blood and the surrounding tissues, and maintenance of this barrier is critical for hemostasis (1). Development of the vasculature is regulated through a number of complex signaling pathways, including Hedgehog, Notch, VEGF, and $\operatorname{TGF} \beta(2,3)$. Formation of stable vasculature additionally requires endothelial-endothelial interactions and interactions among endothelium, pericytes, and surrounding parenchyma (2); however, the signaling pathways involved in these processes are not well understood.

There is considerable overlap between the genes implicated in vascular development in mammals and zebrafish $(4,5)$. In addition, the embryonic transparency and external development of zebrafish embryos make this organism well suited for the study of vascular development and the identification of novel vascular disease genes (6). Toward this end, the first large-scale zebrafish mutagenesis screen for cardiovascular phenotypes identified five hemorrhagic mutants (mush for brains, bubblehead, migraine, gridlock, and leaky heart) (7). The positional cloning of the first of these mutants, gridlock, identified a homolog of HEY2 that is a critical factor in arterial/venous specification $(8,9)$. Subsequently, zebrafish vascular defects due to mutations in genes of the Notch, VEGF, and TGF $\beta$ pathway have been identified ( 8 , 10, 11).

We have identified a recessive zebrafish mutant, termed redhead, which displays cerebral hemorrhage between 2 and 3 days postfertilization (dpf) because of a mutation in p21activated kinase $2 a$ (pak2a), a member of the p21-activated kinase gene family. PAKs, of which there are six family members in mammals, are kinases that act downstream of Rho-family GTPases and regulate a number of diverse biological processes that include immune function, apoptosis, stem cell self-renewal, cell motility, and cytoskeletal rearrangements $(12,13)$. Alterations in PAK function in vitro have been shown to affect endothelial contractility, retraction, and migration (14-17). PAKs are activated by the growth factors basic fibroblast growth factor and VEGF and have been implicated in angiogenesis (15, 18 ) and formation of focal adhesions $(19,20)$; however, there is very limited information available about the functions of PAK proteins in vivo (21).

\section{Results}

rhd Is Characterized by CNS Hemorrhage and Maps to Zebrafish Chromosome 2. We identified a recessive zebrafish mutant, $r h d^{m i 149}$, in a three-generation chemical mutagenesis screen (22) by observation of CNS hemorrhage between 2 and $3 \mathrm{dpf}$ in otherwise morphologically normal-appearing embryos (Fig. 1A). Once hemorrhage occurs, some embryos resolved the hemorrhage and survived, whereas others died. In heterozygous intercrosses, $\approx 10 \%$ of embryos developed hemorrhage, rather than the expected $25 \%$ for a fully penetrant recessive mutation. Thus, the rhd mutant demonstrates incomplete penetrance of the phenotype $(\approx 40 \%)$. Adult homozygotes were viable and fertile, although there was statistically significant loss of $r h d / r h d$ animals by 3 months of age. Genotyping of 262 adult fish revealed 81 $+/+, 134 \mathrm{rhd} /+$, and $47 \mathrm{rhd} / \mathrm{rhd}(P<0.015)$.

The rhd gene was mapped by bulked segregant analysis with a panel of 300 short sequence-length polymorphism markers spanning the zebrafish genome to identify markers that cosegregated with the hemorrhagic phenotype. By this analysis, mul-

Author contributions: D.A.B., F.S., J.A.S., D. Ginsburg, and S.E.L. designed research; D.A.B F.S., J.S.Y., M.K., J.A.S., L.K.B., B.M., S.K., and A.W.H. performed research; M.K., J.D.A., P.J., D. Goldman, N.D.L., and B.M.W. contributed new reagents/analytic tools; D.A.B., F.S., M.K., J.A.S., P.J., P.A.R., B.M.W., D. Ginsburg, and S.E.L. analyzed data; and D.A.B., J.A.S., B.M.W., D. Ginsburg, and S.E.L. wrote the paper.

The authors declare no conflict of interest.

This article is a PNAS Direct Submission.

Abbreviations: dpf, days postfertilization; hpf, hours postfertilization; PAK, p21-activated kinase; CCM, cerebral cavernous malformation; MO, morpholino.

†Present address: Department of Genetics, Case Western Reserve University, Cleveland, $\mathrm{OH}$ 44106

§\о whom correspondence should be addressed at: University of Michigan, 210 Washtenaw Avenue, Ann Arbor, MI 48109-2216. E-mail: ginsburg@umich.edu.

This article contains supporting information online at www.pnas.org/cgi/content/full/ 0700947104/DC1.

() 2007 by The National Academy of Sciences of the USA 
A

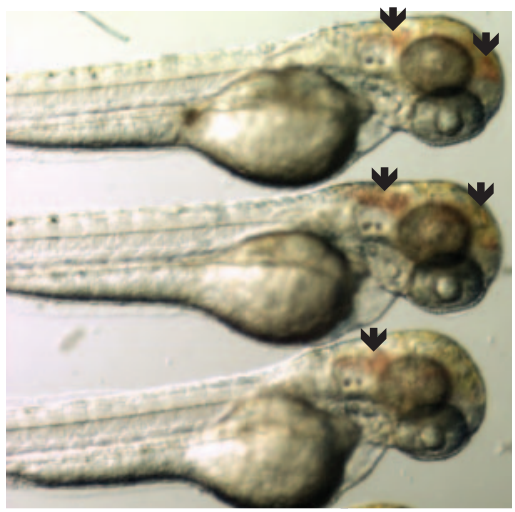

B
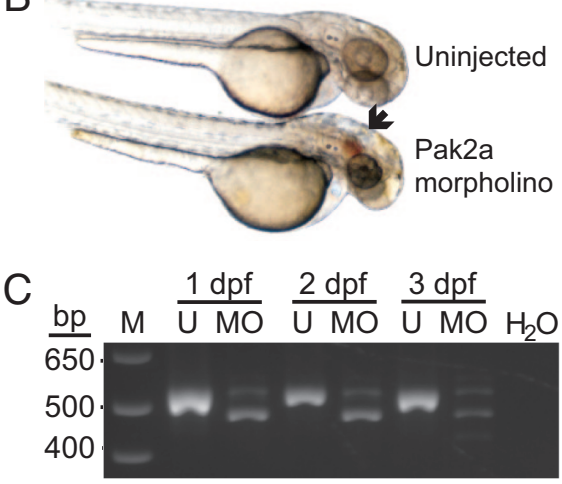

Fig. 1. Cerebral hemorrhage in rhd embryos. (A) Three rhd embryos at $3 \mathrm{dpf}$ in lateral views. Arrows indicate area of hemorrhage. $(B)$ Injection of a pak2a $\mathrm{MO}$ causes cerebral hemorrhage similar to that seen in $r$ d. Negative control and pak2a MO-injected embryo at $3 \mathrm{dpf}$ are shown. (C) pak2a RT-PCR of RNA isolated from embryos injected with pak2a MO show aberrant transcripts.

tiple markers on chromosome 2 were linked to rhd (z1484, z99202, z22747, z13475, z13521, and z7358). High-resolution genetic mapping of $r h d$ was performed by genotyping 729 mutant embryos with additional SNPs and short sequence-length polymorphisms [supporting information (SI) Table 2]. The rhd nonrecombinant interval, defined by two recombinant animals, encompassed $<100 \mathrm{~kb}$ of genomic sequence (Fig. $2 A$ ). Analysis of this genomic interval identified seven candidate genes: pdcd10-like, golph4-like, ccnl1, pak2a, ptx3-like, klhl6, and ing5b (Fig. 2B).

The rhd pak2a Gene Contains a Splice-Donor Site Variant. Priority was given to pdcd10-like, golph4-like, ccnl1, and pak2a for further characterization. Of particular interest was pdcd10-like because mutations in the human ortholog, PDCD10, have been identified in patients with cerebral cavernous malformation (CCM) (23). However, no nonsynonymous coding changes were found in $r h d$. Surprisingly, direct sequence comparison between WT and mutant embryos identified splice-donor site variants in both golph4-like (IVS13: G+1T) and pak2a (IVS9: T+2A). RT-PCR confirmed aberrant premRNA splicing in both genes (Fig. 2 $C-E$ ). The two fish defining the rhd nonrecombinant interval out of 1,458 informative meioses were genotyped for both of these SNPs by direct sequencing. Whereas the pak $2 a$ variant was still nonrecombinant with the rhd phenotype, the golph4-like variant and pdcd10-like were outside of the refined nonrecombinant interval located between D2Umi17 and D2Umi18 (Fig. 2B). The pak $2 a$ variant was not present in five fish from each of the strains $\mathrm{EK}$, WIK, AB, and TL, suggesting that it is not a common polymorphism among laboratory strains.
Full-length pak2a encodes 517 amino acids in 14 exons. The splice site variant in pak2a causes skipping of exon 9 and encodes a predicted protein of 272 amino acids, which truncates 250 $\mathrm{C}$-terminal amino acids and includes five unique residues at the $\mathrm{C}$ terminus (Fig. $2 C$ ). This truncation removes nearly the entire kinase domain (Fig. $2 F$ ) and is predicted to be nonfunctional based on the loss of activity that has been reported because of missense mutations in the kinase domains of several PAK homologs (24-26).

By fluorescent RT-PCR analysis, the exon 9-skipped isoform accounts for $>90 \%$ of the pak2a transcripts in $r h d$ homozygotes (Fig. 2G). Three other splice variants were identified that used cryptic splice donors and together account for the remaining $\approx 10 \%$ of transcripts (Fig. $2 H$ ). Two of these, pak $2 a^{314}$ and $\mathrm{p} a k 2 a^{294}$, disrupt the ORF and encode truncated proteins of 314 amino acids (10 unique) and 294 amino acids ( 5 unique), both again removing most of the kinase domain. The third splice variant, pak $2 a^{S 305 R N}$, contains an additional three nucleotides, substituting $\mathrm{R}$ for $\mathrm{S} 305$ followed by an $\mathrm{N}$ insertion, which maintains the reading frame and preserves an intact kinase domain. However, the S305 position is invariant among Pak2a homologs from 10 other species (SI Fig. 6), consistent with an important functional role for this residue, suggesting that Pak2a (S305RN) may also exhibit decreased or absent activity.

rhd Is Caused by a Mutation in pak2a. To confirm that a mutation in pak $2 a$ is responsible for the $r h d$ phenotype, 25 or $250 \mathrm{ng} / \mu \mathrm{lWT}$ pak $2 a$ mRNA or GFP mRNA was injected into one- to four-cell embryos from an rhd heterozygous intercross. Injection of 25 $\mathrm{ng} / \mu \mathrm{l}$ pak2a mRNA had no effect on the $r h d$ phenotype (data not shown); however, injection of $250 \mathrm{ng} / \mu \mathrm{l}$ pak2a significantly reduced the frequency of hemorrhage relative to controls. Fifty-five bleeders were identified among 665 GFP injected embryos $(8.3 \%)$, which is similar to the penetrance in uninjected embryos. In contrast, only eight bleeders were identified among 731 pak $2 a$ mRNA-injected embryos $(1.1 \%)\left(P<1 \times 10^{-11}\right.$ compared with GFP injection). Thus, pak2a mRNA rescues the hemorrhagic phenotype in rhd embryos.

We next tested whether knockdown of Pak2a would mimic the rhd phenotype. Injection of a pak2a morpholino (MO) into WT embryos phenocopies the embryonic cerebral hemorrhage of rhd, although generally with greater severity as evidenced by displacement of all circulating red cells into the hemorrhage site and hydrocephalus (Fig. 1B). Analysis of the splice products from MO-injected and control embryos showed aberrant splicing in MO-injected fish (Fig. $1 C$ ). Injection of the pak2a MO resulted in $\approx 80 \%$ of embryos with hemorrhage but little general lethality. Taken together, these experiments demonstrate that rhd is caused by a mutation in pak2a. These observations also suggest that the rhd mutation may be hypomorphic, perhaps because of a low level of residual Pak2a activity from the minor pak $2 a^{S 305 R N}$ splice variant.

Pak2a Kinase Activity Is Required for Vascular Integrity. To test whether the kinase activity of Pak2a was required for the downstream signaling events that regulate proper vascular function in vivo, we generated a "kinase-dead" allele of Pak2a containing a K271R mutation (SI Fig. 6) that is homologous to mutations that disrupt the kinase function of human PAK1 (K299R) and PAK2 (K278R) (27, 28). K271R or WT pak2a mRNA was coinjected with the pak2a MO into WT embryos and bleeding was scored at $3 \mathrm{dpf}$ (SI Table 3 ). The pak2a MO injected alone resulted in 92 bleeders of 114 total embryos $(81 \%)$ Coinjection of the kinase-dead K271R pak2a mRNA resulted in 73 bleeders of 92 total embryos (79\%), which was not statistically different from the MO-alone injection $(P>0.7)$, whereas coinjection of pak $2 a$ WT mRNA resulted in 67 bleeders of 128 total embryos $(52 \%)$, again demonstrating rescue with WT 
A

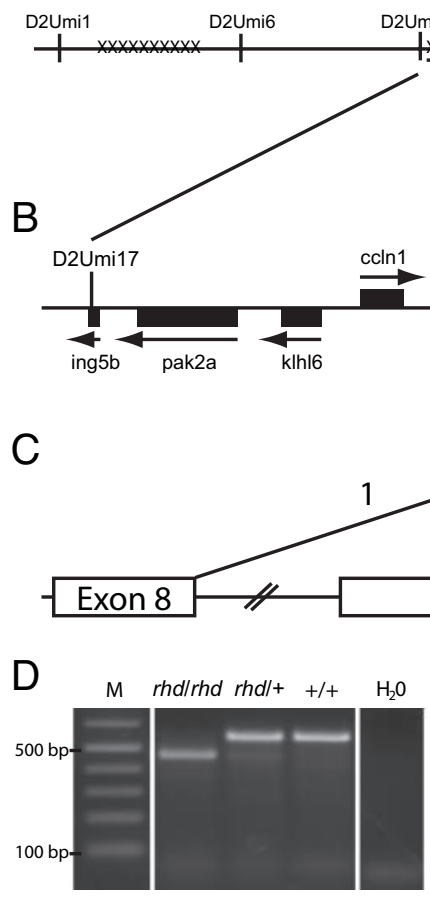

E

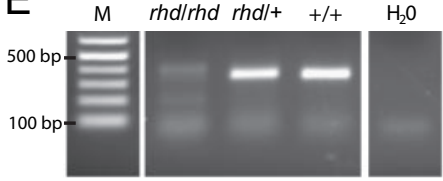

$\mathrm{F}$

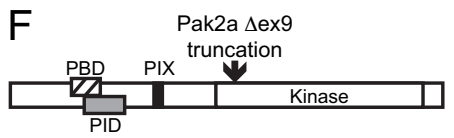

Q 21 binding domain

$\square$ Pak autoinhibitory domain

$\beta$-pix interacting domain

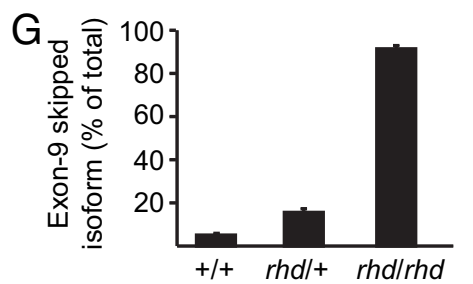

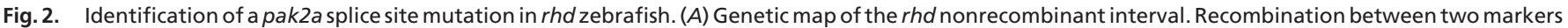

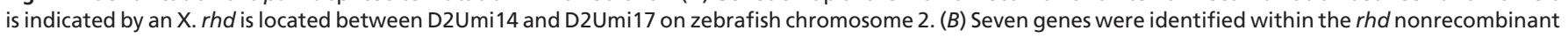

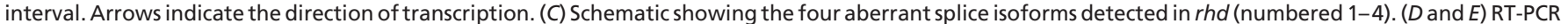

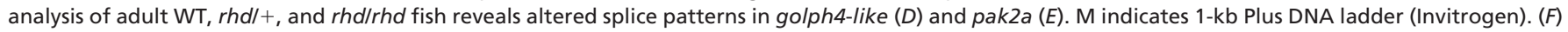

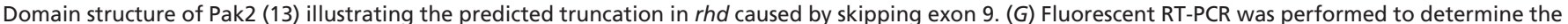

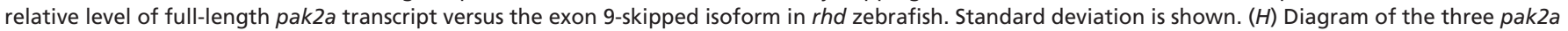

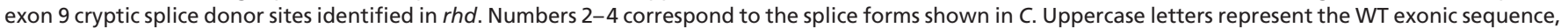
whereas lowercase letters represent the WT intronic sequence. $n$, number of clones among eight that were sequenced.

Pak2a $\left(P<1 \times 10^{-13}\right.$ compared with MO-alone injection). The pak2a K271R mRNA was similarly unable to rescue the bleeding in $r h d$ homozygous embryos $(P>0.8$ compared with uninjected $)$, in contrast to pak2a WT mRNA, which was able to rescue the hemorrhage $(P<0.0008)$ (SI Table 3$)$. These data demonstrate that the kinase function of Pak2a, presumably acting on a downstream substrate, is required for the maintenance of vascular integrity in vivo.

Two Pak2 Orthologs, Pak2a and -2b, Have Overlapping Functions. Although there is only a single $P A K 2$ gene in mammals, examination of the zebrafish genome revealed two $P A K 2$ orthologs, which we have termed pak2a and pak $2 b$. There are numerous examples of duplicated genes throughout the zebrafish genome that appear to be the result of a teleost-specific complete genome duplication event (29). pak2a is on chromosome 2 and is the gene mutated in $r h d$, whereas pak2b is located on zebrafish chromosome 22 (Ensembl Zv6). Each zebrafish PAK2 ortholog has a neighboring ortholog of ING5 (ing $5 a$ and ing $5 b$ ), thus demonstrating conserved synteny between the two chromosomes. Pak2a and Pak2b proteins are $84 \%$ identical with each other, whereas Pak2a is $85 \%$ and Pak2b is $82 \%$ identical with human PAK2 (SI Fig. 6). Within the kinase domain, Pak2a and Pak2b are $98 \%$ identical.
Whole-mount in situ hybridization for pak $2 a$ and pak $2 b$ showed that both were widely expressed at low levels, with more prominent expression in the head at 36 hours postfertilization (hpf) (Fig. 3) and $48 \mathrm{hpf}$ (data not shown). To further examine the expression of these genes, in situ hybridization was performed on tissue sections from 48-hpf embryos. The expression of pak $2 a$ and pak $2 b$ demonstrated significant overlap. pak $2 a$ was widely expressed throughout the brain and spinal cord as well as the epidermis and endoderm (Fig. $3 E$ and $F$ ). pak $2 b$ was also expressed broadly in the brain, spinal cord, and endoderm but was not detected in the epidermis (Fig. $3 H$ and $I$ ). pak2a and pak $2 b$ endothelial cell expression was also detected in sections of the dorsal aorta (Fig. $3 G$ and $J$ ).

To assess the function of Pak2b in vivo, an MO against pak $2 b$ was used that targets the splice junction of exon 5. The resulting pak2b transcript skips exon 5 , thereby disrupting the reading frame, and is predicted to encode a truncated, nonfunctional protein. In contrast to the pak $2 a \mathrm{MO}, \mathrm{WT}$ embryos injected with a pak $2 b$ MO displayed no abnormal phenotype (Fig. 4). Similar levels of exon skipping were observed with both the pak2a and pak $2 b$ MOs (data not shown), suggesting that Pak2b is not required for early vascular integrity, although it is possible that a low level of residual Pak2b activity may be sufficient.

The pak $2 b$ MO was also injected into embryos from an rhd 
A
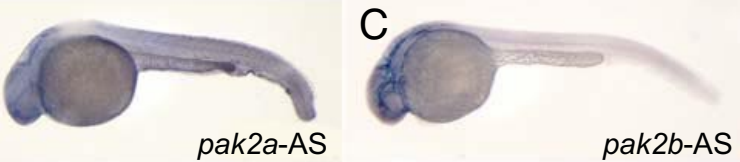

B

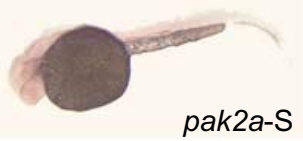

$\mathrm{D}$

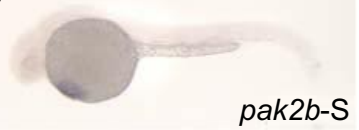

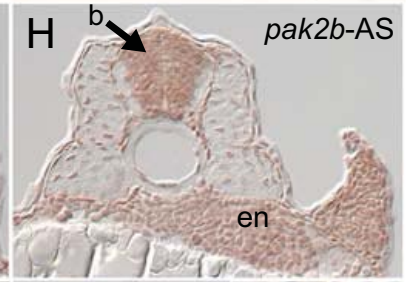

$\mathrm{F}$

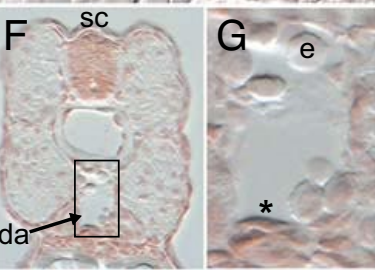

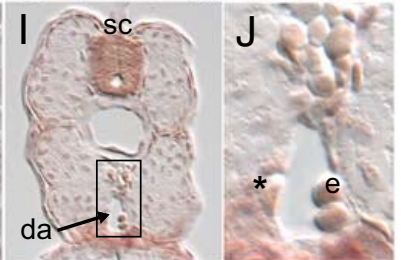

Fig. 3. In situ hybridization with pak2a and pak2b. (A-D) Lateral views with heads to the left of 36-hpf embryos hybridized with pak2a antisense $(A)$, pak2a sense $(B)$, pak $2 b$ antisense $(C)$, or pak $2 b$ sense $(D)$ probes. $(E-J)$ In situ hybridizations of cryosectioned 48-hpf embryos. Staining with NBT/BCIP is shown by a brownish-pink color. (E-G) pak2a antisense. $(H-J)$ pak2b antisense. The areas shown by a rectangle in $F$ and $I$ are enlarged in $G$ and $J$, respectively. The asterisks in $G$ and $J$ label endothelial cells. b, brain; ep, epidermis; en, endoderm; da, dorsal aorta; sc, spinal cord; e, erythrocyte.

heterozygote intercross to determine the effect of Pak2b knockdown not only in WT embryos but in rhd heterozygotes and homozygotes as well (Fig. 4 and SI Table 4). Consistent with previous results, no phenotype was observed in the WT embryos. However, in $r h d /+$ heterozygotes, injection with the pak2b MO caused CNS hemorrhage, similar to the rhd homozygous phenotype. Furthermore, injection of pak2b MO into rhd/rhd homozygotes resulted in CNS hemorrhage of greater severity than observed in rhd/rhd homozygotes alone, accompanied by ventricular swelling, pericardial edema, embryonic lethality, and an increase in the penetrance of hemorrhage. These results suggest an overlap in function between Pak2a and Pak2b. Interestingly, pak $2 b$ mRNA was not able to rescue rhd (SI Table 5), consistent with the observation that endogenous $p a k 2 b$ is expressed in the CNS but does not prevent the rhd phenotype.

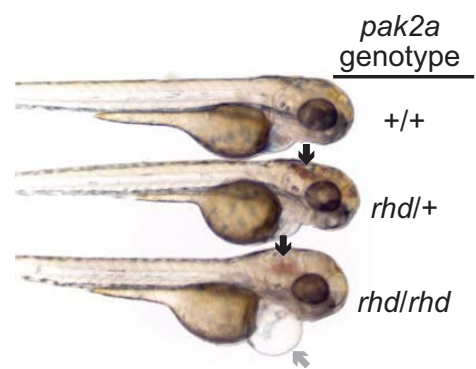

Fig. 4. Pak2b deficiency modifies the severity of hemorrhage in rhd. Representative pak2 $b \mathrm{MO}$-injected embryos of each pak2a genotype are shown at $3 \mathrm{dpf}$ with black arrows showing hemorrhage and the gray arrow indicating pericardial edema.
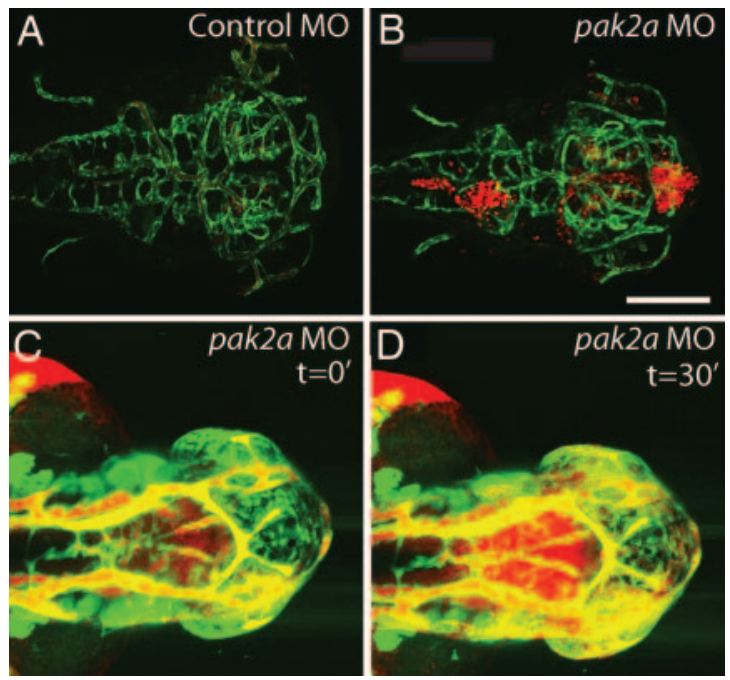

Fig. 5. Normal vascular patterning in Pak2a-deficient animals. ( $A$ and $B$ ) Confocal imaging of 2-dpf Tg(flk1:EGFP);Tg(gata1:dsred) homozygous double transgenic animals injected with either control $\mathrm{MO}(A)$ or pak2a $\mathrm{MO}(B)$. Animals injected with pak2a MO display severe hemorrhage, but their vascular pattern does not differ significantly from control MO-injected animals. ( $C$ and $D$ ) Confocal imaging of $\approx 2$-dpf Tg(flki-:EGFP) transgenic embryos injected with pak2a MO. Shown is an embryo that had CNS hemorrhage and was injected with quantum dots $(2 \mu \mathrm{m})$. $C$ is at time point 0 , and $D$ is after $30 \mathrm{~min}$, when leakage has occurred. All images are dorsal views, head to the right. (Scale bar, $200 \mu \mathrm{m}$.)

pak2a Morphants Have Normal Vessel Patterning and Hemostatic Function and Are Rescued by Endothelial-Specific Pak2a. Analysis of in vivo thrombus formation by using a pulsed-dye laser injury model (30) failed to detect any difference in the time to thrombotic vascular occlusion, suggesting that the CNS hemorrhage in Pak2a-deficient animals is also not the result of a global defect in hemostasis (SI Fig. 7). We next used a double transgenic $\operatorname{Tg}(\mathrm{flk1}$ :EGFP); $\operatorname{Tg}($ gata1:dsred) line to simultaneously assess vascular pattern (green fluorescent vessels) and vascular integrity (red fluorescent erythrocytes) $(31,32)$. Massive cranial hemorrhage was readily apparent in confocal images of pak $2 a$ MO-injected animals compared with control MO-injected animals (Fig. $5 A$ and $B$ ). Large numbers of extravasated erythrocytes were found in the hindbrain ventricle, which appeared enlarged in many morphants. Fluorescent erythrocytes were also present in the more rostral ventricles as well as in nonventricular cranial interstitial spaces. Despite the severe hemorrhage, the pattern of both major primary vasculogenic vessels (e.g., primordial hindbrain channel, basilar artery, etc.) and secondary angiogenic vessels was not significantly different between control and pak2a MO-injected animals. Thus, loss of Pak2a leads to a specific defect in vascular integrity without affecting vascular patterning.

To further examine vessel integrity, 30- to 36-hpf $\mathrm{Tg}$ (flk1:EGFP) embryos that were previously treated with pak2a or control MO were intravascularly injected with $\approx 0.02-\mu \mathrm{m}$ sized QD605 red fluorescent quantum dots before the appearance of an overt hemorrhage. Because of their small size, leakage of the quantum dots should provide a more sensitive indication of a vascular wall defect or increased permeability than extravasation of much larger $(\approx 8-\mu \mathrm{m})$ erythrocytes. After injection, embryos were observed by multiphoton time lapse imaging to determine the timing and location of leakage. No more leakage of the quantum dots in pak2a morphants was observed than in control animals until the appearance of overt hemorrhage, after which quantum dots quickly spread into regions of the brain and ventricles adjacent to the hemorrhage site (Fig. $5 C$ and $D$ ). Thus, 
Table 1. Rescue of $r$ hd by endothelial-specific expression of pak2a

\begin{tabular}{lcccc} 
Transgene & $\begin{array}{c}\text { No. of } \\
\text { bleeders }\end{array}$ & $\begin{array}{c}\text { No. of } \\
\text { nonbleeders }\end{array}$ & Total & $P$ value \\
\hline Fli-GFP & $153(48)$ & 164 & 317 & \\
Fli-pak2a & $35(14)$ & 211 & 246 & $1 \times 10^{-36}$
\end{tabular}

Number in parentheses represent the percentage of the total.

Pak2a-deficient blood vessels appear to be prone to rupture and catastrophic loss of junctional integrity, without evidence for a general increase in leakiness or permeability. These results are consistent with preliminary electron microscopic studies, which failed to detect an overt defect in endothelial junctions (data not shown).

To directly examine the role of endothelial Pak2a in the pathogenesis of CNS hemorrhage, pak $2 a$ or an egfp control was transiently expressed under the control of the endothelial-cellspecific fli enhancer/promoter flanked by Tol2 transposable elements (N.D.L., unpublished data). Coinjection of fli-pak2a with the pak $2 a \mathrm{MO}$ and transposase resulted in significant rescue of the hemorrhagic phenotype (Table 1). Examination of fli-egfp embryos revealed mosaic vascular expression in 54\% of embryos. Taken together, these data suggest that the loss of vascular integrity in the CNS of rhd is due to a cell-autonomous defect in endothelial cell function.

\section{Discussion}

We have identified a mutation in pak $2 a$ responsible for CNS hemorrhage in the rhd zebrafish mutant. Paks have previously been shown to act downstream of basic fibroblast growth factor and integrins (18) during angiogenesis and to bind the adaptor protein, NCK, an interaction required for angiogenesis (15) and the correct formation of focal adhesions $(19,20)$. PAK2 has also been shown to induce retraction of endothelial cells (17). Our data provide direct in vivo evidence that Pak2a function within the endothelial cell is specifically required for the formation of a stable vasculature, at least in a subset of vessels. Recent in vitro data have shown that PAK is involved in increasing vascular permeability in response to VEGF or other physiological signals $(14,16)$. We hypothesize that the function of PAKs in the formation of vessels (angiogenesis) is different from its role in regulating endothelial cell permeability in already formed vessels.

Deficiency of the single mammalian PAK2 results in early embryonic lethality (13). In the zebrafish, the presence of two orthologs of PAK2 may have allowed us to dissect the critical role of Pak2 in the control of CNS vascular integrity in vivo, without the widespread defects seen in mammals (13). The finding that loss of Pak $2 \mathrm{~b}$ increases the severity of the phenotype in rhd mutants suggests an overlapping function in the vasculature for these two paralogs. However, loss of Pak2b by itself does not cause hemorrhage, and pak $2 b$ overexpression fails to rescue the rhd phenotype. The $98 \%$ sequence identity between the Pak2a and Pak2b kinase domains suggests that key differences in other domains are critical for Pak2 function in vivo.

The inability of a kinase-dead allele of Pak2a to rescue rhd or the Pak2a morphant phenotype demonstrates that phosphorylation by Pak2 is required by the relevant downstream targets to maintain vascular integrity. Further evidence for the importance of downstream Pak2 signaling comes from the identification of $\beta$-pix as the gene mutated in another CNS hemorrhage zebrafish mutant, bubblehead (33). After the formation of a complex with $\beta$-pix, Pak2 is shuttled to a subsequent interaction with Rac1 and Cdc42, which phosphorylate and thereby activate Pak2 (34).
The CNS hemorrhage phenotypes of rhd and bubblehead zebrafish suggest that mutations altering the Pak $2 / \beta$-pix pathway may contribute to vascular and/or hemorrhagic disorders in humans. The hemorrhage specifically in the CNS may be due to the high expression of Pak2a in the CNS or may reflect specific properties in the CNS that make the vasculature more susceptible to the damage. In humans, the genetic disorder CCM is characterized by vascular lesions localized to the CNS. The most common form of CCM (CCM1) is due to mutations in KRIT1 (35), which interacts with CCM2 (the gene mutated in CCM2), with both proteins hypothesized to play a role in endothelial cell signal transduction via the p38 MAPK pathway (36). Because PAK2 has been implicated in the activation of the p38 signaling cascade (37), defects in the Pak $2 / \beta$-pix pathway could account for additional, unexplained cases of CCM or could function as modifier genes in these disorders or in clinical settings such as CNS hemorrhage in premature infants. Further analyses of this mutant and identification of additional mutants will provide insights into the critical pathways for vascular integrity.

\section{Materials and Methods}

Animals. Zebrafish (Danio rerio) were raised as described (38) in accordance with animal care guidelines. Michigan pet store fish were used for the mutagenesis and crosses were performed with TL for meiotic mapping. A doubly homozygous $\operatorname{Tg}(f l k 1: E G F P) ; \operatorname{Tg}$ (gata1:dsred) line was used for simultaneous imaging of vascular pattern and vascular integrity $(31,32)$.

Genotyping. rhd embryos were genotyped with microsatellite markers D2Umi5 and D2Umi11 (SI Table 2), which flank the pak2a IVS9: $\mathrm{T}+2 \mathrm{~A}$ mutation. When a recombination was detected between the two flanking markers, pak $2 a$ intron 9 was directly PCR amplified with primer pair E (SI Table 2) and digested with the restriction enzyme SphI (New England Biolabs, Beverly, MA), which distinguishes rhd and WT embryos based on the IVS9:C+44T SNP located 42 bp from the IVS9: $\mathrm{T}+2 \mathrm{~A}$ mutation. All D2Umi markers described in this study (SI Table 2) are located on chromosome 2 and were developed at the University of Michigan.

Embryo Injection Experiments. pak2a mRNA was generated from IMAGE clone 6997095 linearized with NgoM IV (New England Biolabs). Site-directed mutagenesis was performed (QuikChange II; Stratagene, La Jolla, CA) to generate the K271R kinase-dead allele of pak2a (SI Materials and Methods). The mMessage mMachine SP6 or T7 kits (Ambion, Austin, TX) were used to generate mRNAs. Embryos were injected into the yolk sac at the one- to four-cell stage with $\approx 1 \mathrm{nl}$ of mRNA at a concentration of $250 \mathrm{ng} / \mu \mathrm{l}$. Embryos were blindly phenotyped for the presence or absence of a bleeding event at $3 \mathrm{dpf}$. Statistical significance was calculated by $\chi^{2}$ analysis.

MOs (GeneTools, Philomath, OR) were designed to block the splice-donor sites after exon 8 in pak $2 a$ and exon 5 in pak $2 b$. The pak $2 a$ MO sequence was 5'-AATAG AGTAC AACAT ACCTC TTGGC-3' and pak2b MO was 5'-AATAA ATGAT ACGTA CCGAC TGTTC-3' . Eight picograms of each MO was injected per embryo.

Endothelial-Specific Rescue. To drive endothelial cell-specific gene expression, a chimeric promoter/enhancer fragment was generated that includes the fli1 promoter and enhancer as well as the Tol 2 element. For rescue experiments, pak $2 a$ and egfp from were placed into the Tol2-fli destination vector (SI Materials and Methods). Transposase mRNA (39) was prepared by using mMessage mMachine (Ambion). One picogram of either Tol2fli pak2a or $g f p$ final circular plasmid was coinjected with both 1 pg of transposase mRNA and 8 pg of pak2a MO into one-cell embryos, and phenotyped at $3 \mathrm{dpf}$ as described above. 
RT-PCR. Total RNA was isolated from adult fish with the RNA Mini Kit (Qiagen, Valencia, CA) and was reverse transcribed by using the iScript cDNA synthesis kit (Bio-Rad, Hercules, CA). golph 4 and pak2 $a$ cDNAs were amplified with primer pairs A and $\mathrm{B}$, respectively (SI Table 2). The relative levels of each pak2a splice isoform were quantitated by fluorescence-based RT-PCR by using established methods (40).

In Situ Hybridization. RNA in situ hybridization was performed as described (40). pak2a probe was generated by using the fulllength pak $2 a$ cDNA in IMAGE clone 6997095 linearized with StuI or NotI and SP6 or T7 RNA polymerase to generate sense and antisense transcripts, respectively. pak $2 b$ probe was generated by using the full-length pak $2 b$ cDNA cloned in pCS2+ linearized with BamHI or NotI and T7 or SP6 RNA polymerase to generate sense and antisense transcripts, respectively. For in situ hybridization of sections, embryos were first cryosectioned followed by in situ hybridization with probes at $4 \mu \mathrm{g} / \mathrm{ml}$ according to established protocol (41).

Microdot Injection and Confocal Microscopy. Confocal microscopy of $\mathrm{Tg}(\mathrm{flk1}$ :EGFP); $\mathrm{Tg}$ (gata1:dsred) animals was performed on an Olympus (Melville, NY) FV1000 confocal microscope with a krypton-argon laser source. Animals were treated with 6-propyl-

1. Mehta D, Malik AB (2006) Physiol Rev 86:279-367.

2. Coultas L, Chawengsaksophak K, Rossant J (2005) Nature 438:937-945.

3. Jain RK (2003) Nat Med 9:685-693.

4. Roman BL, Pham VN, Lawson ND, Kulik M, Childs S, Lekven AC, Garrity DM, Moon RT, Fishman MC, Lechleider RJ, et al. (2002) Development (Cambridge, UK) 129:3009-3019.

5. Mably JD, Chuang LP, Serluca FC, Mohideen MA, Chen JN, Fishman MC (2006) Development (Cambridge, UK) 133:3139-3146.

6. Zhong TP (2005) in Current Topics in Developmental Biology (Academic, New York), pp 53-81.

7. Stainier DY, Fouquet B, Chen JN, Warren KS, Weinstein BM, Meiler SE, Mohideen MA, Neuhauss SC, Solnica-Krezel L, Schier AF, et al. (1996) Development (Cambridge, UK) 123:285-292.

8. Zhong TP, Childs S, Leu JP, Fishman MC (2001) Nature 414:216-220.

9. Zhong TP, Rosenberg M, Mohideen MA, Weinstein B, Fishman MC (2000) Science 287:1820-1824.

10. Habeck H, Odenthal J, Walderich B, Maischein H, Schulte-Merker S, Tubingen 2000 Screen Consortium (2002) Curr Biol 12:1405-1412.

11. Jadrich JL, O'Connor MB, Coucouvanis E (2006) Development (Cambridge, UK) 133:1529-1541.

12. Benitah SA, Frye M, Glogauer M, Watt FM (2005) Science 309:933-935.

13. Hofmann C, Shepelev M, Chernoff J (2004) J Cell Sci 117:4343-4354.

14. Gavard J, Gutkind JS (2006) Nat Cell Biol 8:1223-1234.

15. Kiosses WB, Daniels RH, Otey C, Bokoch GM, Schwartz MA (1999) J Cell Biol 147:831-844

16. Stockton RA, Schaefer E, Schwartz MA (2004) J Biol Chem 279:46621-46630.

17. Zeng Q, Lagunoff D, Masaracchia R, Goeckeler Z, Cote G, Wysolmerski R (2000) J Cell Sci 113:471-482.

18. Hood JD, Frausto R, Kiosses WB, Schwartz MA, Cheresh DA (2003) J Cell Biol 162:933-943.

19. Stoletov KV, Gong C, Terman BI (2004) Exp Cell Res 295:258-268.

20. Stoletov KV, Ratcliffe KE, Spring SC, Terman BI (2001) J Biol Chem 276:22748-22755.

21. Hofmann C, Shepelev M, Chernoff J (2004) J Cell Sci 117:4343-4354.

22. Gulati-Leekha A, Goldman D (2006) Dev Biol 296:29-47.

23. Bergametti F, Denier C, Labauge P, Arnoult M, Boetto S, Clanet M, Coubes $\mathrm{P}$, Echenne B, Ibrahim R, Irthum B, et al. (2005) Am J Hum Genet 76:42-51. 2-thiouracil to inhibit pigment formation and mounted for imaging in 2\% agarose in Danieu's solution with tricaine/MS-222 as per published methods (38). Image stacks were collected with 5 - $\mu \mathrm{m}$ spacing between planes at $1,024 \times 1,024$ or $512 \times 512$ pixel resolution. Image rendering from collected image stacks was performed with Olympus Fluoview software (41). For leakage experiments, 0.02- $\mu \mathrm{m}$-sized QD605 red-fluorescent quantum dots (Invitrogen, Carlsbad, CA) were injected into the sinus venosus of 30-36 hpf $\mathrm{Tg}(\mathrm{flk1}$ :EGFP) embryos (42).

We thank Dr. D. Turner (University of Michigan) for the gift of the pCS2+ GFP plasmid and Drs. R. Kaufman and A. Saltiel for helpful discussions. We thank C. Cooke, T. Melendez, J. Manueland, and members of the Unit for Laboratory Animal Medicine staff for zebrafish husbandry. We also thank Dr. D. Traver (University of California at San Diego, La Jolla, CA) and Dr. L. Zon (Children's Hospital, Boston, MA) for the gata:dsRed line and Dr. J.-N. Chen (University of California, Los Angeles, CA) for the Flk:GFP line, as well as Dr. B. Roman (University of Pittsburgh, Pittsburgh, PA) for the combined cross. This work was supported by the National Hemophilia Foundation (D.A.B.); the V Foundation (S.E.L.); National Institutes of Health Grants F32HL82213-01 (to D.A.B.), K22-CA098024 (to S.E.L.), R37-HL039693 (to D. Ginsburg), P01-HL057346 (to D. Ginsburg), R01-HL077910 (to P.J.); and Michigan Economic Development Corporation, Life Sciences Corridor, Grant MEDC 38 (to D. Goldman and S.E.L.). D. Ginsburg is a Howard Hughes Medical Institute Investigator.

24. Li W, Guan KL (2004) J Biol Chem 279:32824-32831.

25. Rousseau V, Goupille O, Morin N, Barnier JV (2003) J Biol Chem 278:39123920.

26. Zhang S, Han J, Sells MA, Chernoff J, Knaus UG, Ulevitch RJ, Bokoch GM (1995) J Biol Chem 270:23934-23936.

27. Jakobi R, Huang Z, Walter BN, Tuazon PT, Traugh JA (2000) Eur J Biochem 267:4414-4421.

28. Rudel T, Bokoch GM (1997) Science 276:1571-1574.

29. Woods IG, Wilson C, Friedlander B, Chang P, Reyes DK, Nix R, Kelly PD, Chu F, Postlethwait JH, Talbot WS (2005) Genome Res 15:1307-1314.

30. Jagadeeswaran P, Gregory M, Day K, Cykowski M, Thattaliyath B (2005) J Thromb Haemost 3:46-53.

31. Jin SW, Beis D, Mitchell T, Chen JN, Stainier DYR (2005) Development (Cambridge, UK) 132:5199-5209.

32. Traver D, Paw BH, Poss KD, Penberthy WT, Lin S, Zon LI (2003) Nat Immunol 4:1238-1246.

33. Liu J, Fraser SD, Faloon PW, Rollins EL, Vom Berg J, Starovic-Subota O, Laliberte AL, Chen J-N, Serluca FC, Childs SJ (2007) Proc Natl Acad Sci USA 104:13990-13995.

34. Manser E, Loo TH, Koh CG, Zhao ZS, Chen XQ, Tan L, Tan I, Leung T, Lim L (1998) Mol Cell 1:183-192.

35. Laberge-le Couteulx S, Jung HH, Labauge P, Houtteville JP, Lescoat C, Cecillon M, Marechal E, Joutel A, Bach JF, Tournier-Lasserve E (1999) Nat Genet 23:189-193.

36. Zawistowski JS, Stalheim L, Uhlik MT, Abell AN, Ancrile BB, Johnson GL, Marchuk DA (2005) Hum Mol Genet 14:2521-2531.

37. Lamalice L, Houle F, Huot J (2006) J Biol Chem 281:34009-34020.

38. Westerfield M (2000) The Zebrafish Book. A Guide for the Laboratory Use of Zebrafish (Danio rerio) (Univ of Oregon Press, Eugene, OR), 4th Ed.

39. Kawakami K, Takeda H, Kawakami N, Kobayashi M, Matsuda N, Mishina M (2004) Dev Cell 7:133-144.

40. Keller MA, Cassel DL, Rappaport EF, McKenzie SE, Schwartz E, Surrey S (1993) PCR Methods Appl 3:32-38.

41. Kamei M, Saunders WB, Bayless KJ, Dye L, Davis GE, Weinstein BM (2006) Nature 442:453-456.

42. Weinstein BM, Stemple DL, Driever W, Fishman MC (1995) Nat Med $1: 1143-1147$. 